\title{
Highly Stable and Selective Tetrazines for the Coordination-Assisted Bioorthogonal Ligation with Vinylboronic Acids
}

\author{
Selma Eising, ${ }^{\dagger}$ Anthonius H. J. Engwerda, ${ }^{\ddagger}$ Xian Riedijk, $^{\dagger}$ F. Matthias Bickelhaupt, ${ }^{*}, \S_{\odot}$ \\ and Kimberly M. Bonger*, ${ }^{*}$
}

${ }^{\dagger}$ Department of Biomolecular Chemistry, and Department of Theoretical Chemistry, Institute for Molecules and Materials, Radboud UniversityHeyendaalseweg 135, 6525 AJ Nijmegen, The Netherlands

${ }^{\S}$ Department of Theoretical Chemistry and Amsterdam Center for Multiscale Modeling (ACMM), De Boelelaan 1083, 1081 HV Amsterdam, The Netherlands

Supporting Information

ABSTRACT: Bioorthogonal reactions are selective transformations that are not affected by any biological functional group and are widely used for chemical modification of biomolecules. Recently, we reported that vinylboronic acids (VBAs) gave exceptionally high reaction rates in the bioorthogonal inverse electron-demand Diels-Alder (iEDDA) reaction with tetrazines bearing a boron-coordinating pyridyl moiety compared to tetrazines lacking such a substituent. In this integrated experimental and theoretical study, we show how the reaction rate of the VBA-tetrazine ligation can be accelerated by shifting the equilibrium from boronic acid to the boronate anion in the reaction mixture. Quantum chemical activation strain analyses reveal that this rate enhancement is a direct consequence of the excellent electron-donating capability of the boronate anion in which the $\pi$ HOMO is pushed to a higher energy due to the net negative potential of this species. We have explored the second-order rate constants of several tetrazines containing potential VBA-coordinating hydroxyl substituents. We observed an increase in rate constants of several orders of magnitude compared to the tetrazines lacking a hydroxyl substituent. Furthermore, we find the hydroxyl-substituted tetrazines to be more selective toward VBAs than toward the commonly used bioorthogonal reactant norbornene, and more stable in aqueous environment than the previously studied tetrazines containing a pyridyl substituent.

\section{INTRODUCTION}

The development of bioorthogonal reactions has advanced tremendously as it allows selective modification of biomolecules without interfering with any naturally occurring biochemical functionality. ${ }^{1-4}$ The tetrazine ligation is one of the most popular bioorthogonal reactions due to its selectivity and high reaction rates. ${ }^{5-8}$ So far, several bioorthogonal reactants have been developed for this inverse electrondemand Diels-Alder (iEDDA) reaction, such as strained alkynes (e.g., bicyclo[6.1.0]nonyne $(\mathrm{BCN})),{ }^{9,10}$ strained alkenes (e.g., trans-cyclooctene (TCO), ${ }^{11,12}$ norbornene, ${ }^{13}$ and cyclopropene), ${ }^{14,15}$ and nonstrained alkenes (e.g., primary alkenes). ${ }^{16,17}$ During our efforts to improve the reaction rates of the slow-reacting nonstrained alkenes, we found that vinylboronic acids (VBAs) show impressive second-order rate constants $\left(k_{2}\right)$ with 3,6-dipyridiyl-s-tetrazines of up to 27 $\mathrm{M}^{-1} \mathrm{~s}^{-1}$ in $5 \% \mathrm{MeOH} / \mathrm{PBS}(\mathrm{pH}=7.4) .{ }^{18}$ The VBAs are easily accessible, biocompatible with cellular components, and suitable for protein modification. Recently, we showed that vinylboronic acids are also suitable for a bioorthogonal application within the living cell. ${ }^{19}$

Boronic acids possess a vacant $\mathrm{p}$ orbital and are mild organic Lewis acids that form the more electron-rich boronate complex after coordination by a Lewis base. In aqueous media, the boronic acids are in equilibrium with their negatively charged boronate anion, although the VBAs have high $\mathrm{p} K_{\mathrm{a}}$ values and therefore reside mainly in the trivalent boronic acid form at physiological $\mathrm{pH}(\mathrm{pH}=7.4)$ (Figure $1 \mathrm{~A}) .{ }^{20}$ Recently, we reported that VBAs are much more reactive toward pyridylsubstituted tetrazines than toward tetrazines lacking this Lewis basic substituent (Figure 1B). ${ }^{18,21}$ We observed that the high reaction rates are caused by coordination of the nitrogen of the pyridyl ring to the boron of the VBA, which favors the reaction due to the induced proximity and the inductive effect. We used this unique reactivity of the VBA to develop two orthogonal tetrazine ligations and perform a simultaneous dual labeling of two proteins containing a VBA and a norbornene moiety. ${ }^{21}$

Herein, we explore the reactivity of the boronic acids and their tetravalent boronate counterparts toward tetrazines in more detail in a combined theoretical and experimental study, based on density functional theory (DFT) and rate measurements as a function of $\mathrm{pH}$. In addition, we discuss the reactivity

Received: June 20, 2018

Revised: July 22, 2018

Published: August 6, 2018 
A<smiles>OB(O)C=Cc1ccccc1</smiles>

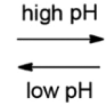<smiles>O[B-](O)(O)C=Cc1ccccc1</smiles>

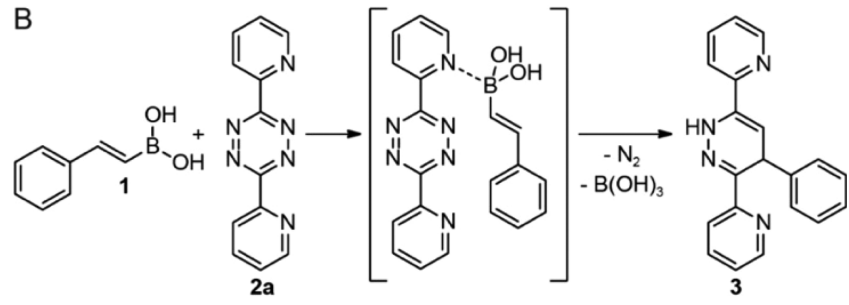

Figure 1. (A) Equilibrium between boronic acid 1a and boronate anion $\mathbf{1 b}$ in aqueous environment. (B) Coordination of the nitrogen of the pyridyl ring of tetrazine $2 \mathrm{a}$ to the boronic acid of VBA $\mathbf{1}$, yielding dihydropyridazine 3 as a single isomer.

of a set of tetrazines bearing conceivable boron-coordinating hydroxyl substituents. We hypothesized that the hydroxyl moiety would favor coordination to the VBA and increase the rate constants of the tetrazine-VBA ligation. Furthermore, we anticipate that orthogonality of these tetrazines toward VBAs could be established as more electron-rich tetrazines react less favorably in the general iEDDA reaction with unsubstituted (strained) alkenes.

\section{RESULTS AND DISCUSSION}

DFT Calculations of the Tetrazine Ligation with VBAs. To provide more insight into the reactivity of the VBA and the boronate anion in the iEDDA reaction with tetrazines, we have explored suitable model reactions using DFT calculations as implemented in the Amsterdam Density Functional (ADF) 2013 software. $^{22}$ For all calculations, the generalized gradient approximation (GGA) was used at the OLYP level. ${ }^{23-25}$ As a basis set, the TZ2P level was used. ${ }^{22}$ Vibrational analyses confirmed zero imaginary frequencies for equilibrium structures and a single imaginary frequency associated with normal mode along the reaction coordinate for transition states.

We first determined the optimized geometries, reaction energies, and transition states of the reaction of vinylboronic acid $\mathbf{6 a}$ with tetrazine $\mathbf{2 b}$ which bears no substituents and cannot coordinate to the boronic acid of the VBA, until the transition state (TS) (Table 1). We compare this reaction to the tetrazine ligations with a nonsubstituted alkene, ethene 4, and with an alkene containing an electron-donating substituent, methylvinylether $\mathbf{5}$. The tetrazine ligation involving methylvinylether $\mathbf{5}$ has a transition state barrier $\left(\Delta E_{\mathrm{TS}}\right)$ that is comparable to that for ethene 4, although both have a lower $\Delta E_{\mathrm{TS}}$ than VBA 6a (Table 1$)$. Also, the reaction energy $\left(E_{\mathrm{r}}\right)$ with ethene $\mathbf{4}$ is more favorable than with VBA $\mathbf{6 a}$, although all reactions are pronouncedly exothermic, with values of -17 , -8.0 , and $-12.8 \mathrm{kcal} \mathrm{mol}^{-1}$ for 4,5 , and $6 \mathrm{a}$, respectively.

Next, we compared the tetrazine ligations of tetrazine $\mathbf{2 b}$ with vinylboronic acid $\mathbf{6 a}$ and the tetracoordinated boronate anion $\mathbf{6 b}$ (Table 1 ). Both $\Delta E_{\mathrm{TS}}$ and $E_{\mathrm{r}}$ are much lower for boronate $\mathbf{6} \mathbf{b}$ than for boronic acid $\mathbf{6 a}$, with $\Delta E_{\mathrm{TS}}+1.6 \mathrm{kcal}$ $\mathrm{mol}^{-1}$ and $E_{\mathrm{r}}$ of $-29.1 \mathrm{kcal} \mathrm{mol}^{-1}$ for $\mathbf{6 b}$, and only $\Delta E_{\mathrm{TS}}$ of $+18.2 \mathrm{kcal} \mathrm{mol}^{-1}$ and $E_{\mathrm{r}}$ of $-12.8 \mathrm{kcal} \mathrm{mol}^{-1}$ for 6 a.

To trace the origin of the enhanced reactivity of $6 \mathbf{b}$ relative to $\mathbf{6 a}$, we have carried out activation-strain analyses (also known as distortion/interaction analyses), ${ }^{26}$ in combination with an analysis of the bonding mechanism between the reactants in the TS based on Kohn-Sham MO theory. ${ }^{27}$ The lower barrier for the boronate anion compared to vinylboronic acid reacting with tetrazine is mainly caused by a more stabilizing interaction energy $\Delta E_{\text {int }}$ in the case of the former. Next, we analyzed the orbital electronic structure of the alkenes 4-6 and tetrazine $\mathbf{2 b}$ (Tables 1 and S3). Figure 2 is a schematic representation of the frontier molecular orbital interactions between VBA $6 \mathrm{a}$ and its boronate anion $\mathbf{6 b}$ with the tetrazine as they emerge from our quantitative KohnSham MO analyses. The dominant bonding mechanism is a donor-acceptor orbital interaction between the occupied C$\mathrm{C} \pi$ bonding orbital of the alkene and the LUMO +1 acceptor orbital of tetrazine $\mathbf{2 b}$. In the case of vinylboronic acid $\mathbf{6 a}$, the

Table 1. Quantum-Chemically Computed Key Parameters of the Ligation of Tetrazine 2b with Alkenes 4-6 ${ }^{a}$

\begin{tabular}{|c|c|c|c|c|c|c|c|c|}
\hline Entry & Alkene & $E_{\mathrm{r}}$ & $\Delta E_{\mathrm{TS}}$ & $\Delta E_{\text {strain }}$ & $\Delta E_{\text {strain-tetrazine }}$ & $\Delta E_{\text {strain-alkene }}$ & $\Delta E_{\text {int }}$ & $\mathrm{HOMO}_{\text {alkene }}$ \\
\hline 1 & $\begin{array}{l}7 \\
4\end{array}$ & -17.1 & +16.9 & +17.1 & +12.6 & +4.5 & -0.23 & -6.51 \\
\hline 2 & $\widehat{\gamma}_{5}^{\prime}$ & -8.0 & +17.9 & +22.4 & +15.0 & +7.5 & -4.5 & -5.09 \\
\hline 3 & $\begin{array}{l}\mathrm{O}^{\prime} \\
6 \mathrm{a}\end{array}$ & -12.8 & +18.2 & +17.7 & +12.2 & +5.5 & +0.5 & -6.57 \\
\hline 4 & 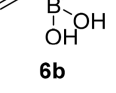 & -29.1 & +1.6 & +15.7 & +11.2 & +4.5 & -14.1 & $-1.05^{[a]}$ \\
\hline
\end{tabular}

${ }^{a}$ Computed at OLYP/TZ2P. Reaction energy $\left(E_{\mathrm{r}}\right)$ and change in transition state $\left(\Delta E_{\mathrm{TS}}\right)$, interaction $\left(\Delta E_{\text {int }}\right)$, and strain energies $\left(\Delta E_{\text {strain }}\right)$ in kcal $\mathrm{mol}^{-1}$ with $\Delta E_{\mathrm{TS}}=\Delta E_{\text {strain }}-\Delta E_{\text {int. }}$ HOMO and LUMO energies in eV. [a] Energy of the HOMO-2, which is the occupied $\pi$ orbital. 


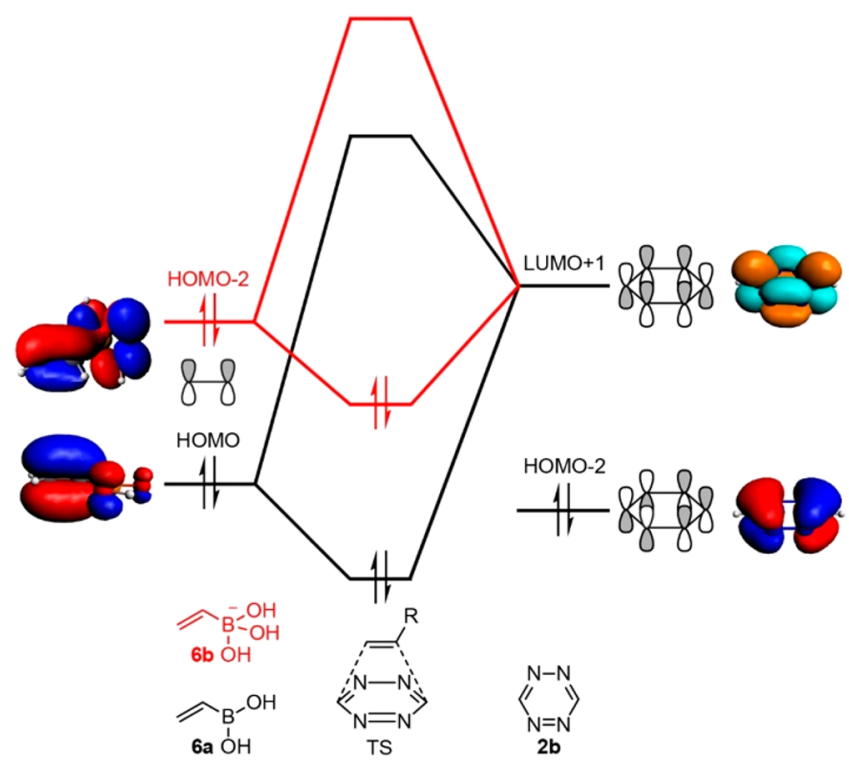

Figure 2. Frontier orbital interactions in the tetrazine ligation. Schematic representation of the frontier molecular orbital interactions of 1,2,4,5-tetrazine $\mathbf{2 b}$ and vinylboronic acid $\mathbf{6 a}$ (black) or vinylboronate $\mathbf{6 b}$ (red) from our quantitative Kohn-Sham MO analyses.

electron-donating $\pi$ orbital is the HOMO whereas for vinylboronate $\mathbf{6 b}$, it is the HOMO-2.

Our analyses reveal that the $\pi$ orbital in boronate $\mathbf{6 b}$ is at higher orbital energy than that in boronic acid 6a, because of the more negative potential in the former, anionic reactant. Consequently, the orbital-energy gap with the tetrazine acceptor orbital is smaller and the interaction $\Delta E_{\text {int }}$ more stabilizing for the boronate anion $\mathbf{6} \mathbf{b}$ than for its boronic acid analogue 6a. Eventually, as pointed out above, this leads to the lower barrier for the reaction involving $\mathbf{6 b}$. Alkenes 4 and 5 have a similar or slightly higher HOMO than vinylboronic acid 6a (Table 1). Therefore, $\mathbf{4}$ and $\mathbf{5}$ should also undergo an iEDDA reaction with tetrazine $\mathbf{2 b}$ (vide infra). Our quantum chemical results agree with our previous finding ${ }^{21}$ that only tetrazines bearing the pyridyl substituent gave high rate constants with VBAs compared to tetrazines lacking a Lewis basic substituent.

pH Effect on the Tetrazine Ligation with VBAs. Next, we investigated whether the lower reaction energy of the boronate anion toward tetrazines could be validated in an experimental setup (Figure 3A). As formation with the boronate anion is favored upon increasing $\mathrm{pH}$, we expected that this would also advance the tetrazine ligation. We examined the reaction of $(E)$-phenylvinylboronic acid $\mathbf{1}$ with 3-phenyl-s-tetrazine 2c, lacking a boron-coordinating atom, between $\mathrm{pH} 8$ and 11. Indeed, the rate of the reaction of VBA 1 with tetrazine $2 \mathrm{c}$ increased at higher $\mathrm{pH}$, indicating that the boronate anion is more reactive than the boronic acid, in accordance with the DFT calculations (Figure 3B). As a control experiment, we examined the reaction of tetrazine $2 \mathrm{c}$ with norbornene $\mathbf{5}$ in the same buffers and observed that the rate is independent of the $\mathrm{pH}$, as expected. In the absence of an alkene, tetrazine $2 \mathrm{c}$ slowly degrades, especially in the basic aqueous solutions (Figure S1).

Second-Order Rate Constants of VBAs with Tetrazines Bearing a Hydroxyl-Substituent. As we established that the tetrazine ligation proceeds faster when the VBA adopts

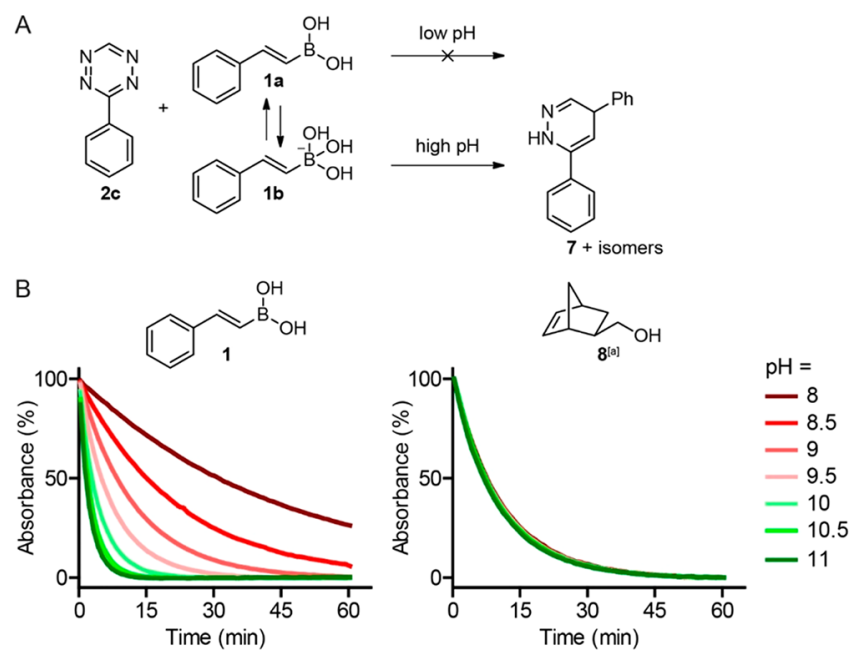

Figure 3. (A) Schematic overview of the $\mathrm{pH}$ effect on the reaction of tetrazine $2 \mathrm{c}$ with VBA 1 yielding dihydropyridazine 7. (B) Reactions of tetrazine 2c with VBA 1 (left) and norbornene 8 (right) in 50\% $\mathrm{MeOH}$ and $50 \% \mathrm{Na}_{2} \mathrm{~B}_{4} \mathrm{O}_{7}$ buffer, ranging from $\mathrm{pH} 8$ until 11 . The reactions were measured at $20{ }^{\circ} \mathrm{C}$ by following the decay of the tetrazine at $540 \mathrm{~nm}$. [a] Endo/exo 2:1.

the boronate anion configuration, we next explored the reactivity of a set of tetrazines bearing hydroxyl substituents. The coordination of the hydroxyl to the VBA could at physiological $\mathrm{pH}$ promote the rate of the iEDDA reaction by making the VBA more reactive and favor the cycloaddition due to the induced proximity. In addition, as the tested hydroxyl substituents are more electron-rich than the pyridine substituent, we expect that the hydroxyl-tetrazines react less favorably in the iEDDA reaction with unsubstituted alkenes, such as norbornene, and thereby become more selective for reaction with VBAs.

We synthesized several tetrazines possessing a hydroxyl substituent, and compared the $k_{2}$ values of the tetrazine ligations of VBA 1 with that of norbornene $\mathbf{8}$ (Figure 4, Table S1). As previously shown, VBA 1 and norbornene 8 have a comparable rate constant of $1.4 \mathrm{M}^{-1} \mathrm{~s}^{-1}$ with dipyridyl-stetrazine $2 \mathrm{a}$ in $50 \% \mathrm{MeOH} / \mathrm{PBS}$ at $20{ }^{\circ} \mathrm{C} .{ }^{18}$ Methyl pyridyl tetrazine $2 \mathrm{e}$ still possesses a boron-coordinating atom, although a 10 -fold lower $k_{2}$ value was found for VBA 1 than for norbornene 8 . Phenyl tetrazine $2 \mathrm{c}$ also showed a lower rate constant for VBA 1 than for norbornene 8. Phenyl methyl tetrazine $2 \mathrm{~h}$, the derivative of $2 \mathrm{e}$ lacking a coordination atom, gives a 100-fold lower rate constant for VBA 1 than for norbornene 8 . Of note, we have previously observed that pyrimidine tetrazines $\mathbf{2 f}$ and $\mathbf{2 g}$ also react poorly with VBAs, which we reasonably ascribe to the lower basicity of the pyrimidine compared to the pyridine substituent. ${ }^{21}$

Introducing an aliphatic hydroxyl substituent, as in tetrazine $2 \mathbf{i}$, resulted in a tremendous increase in reaction rate compared to the nonhydroxyl-containing tetrazine $\mathbf{2 h}$, giving a $k_{2}$ value of VBA 1 with $2 \mathbf{i}$ that is almost 500 -fold higher than with $\mathbf{2 h}$. The rate constant of norbornene $\mathbf{8}$ with tetrazine $\mathbf{2 i}$ slightly dropped compared to the constant with $\mathbf{2 h}$ and was 10 -fold lower than the $k_{2}$ of $2 \mathbf{i}$ with VBA, demonstrating that this hydroxylated tetrazine is more selective toward vinylboronic acids. The reaction of VBA 1 with disubstituted hydroxyethylsubstituted tetrazine $2 \mathbf{j}$ and the hydroxyethyl-methyl-substituted tetrazine $2 \mathbf{k}$ showed lower rate constants than with tetrazine $\mathbf{2} \mathbf{i}$, possibly due to lack of an aromatic substituent on 
A
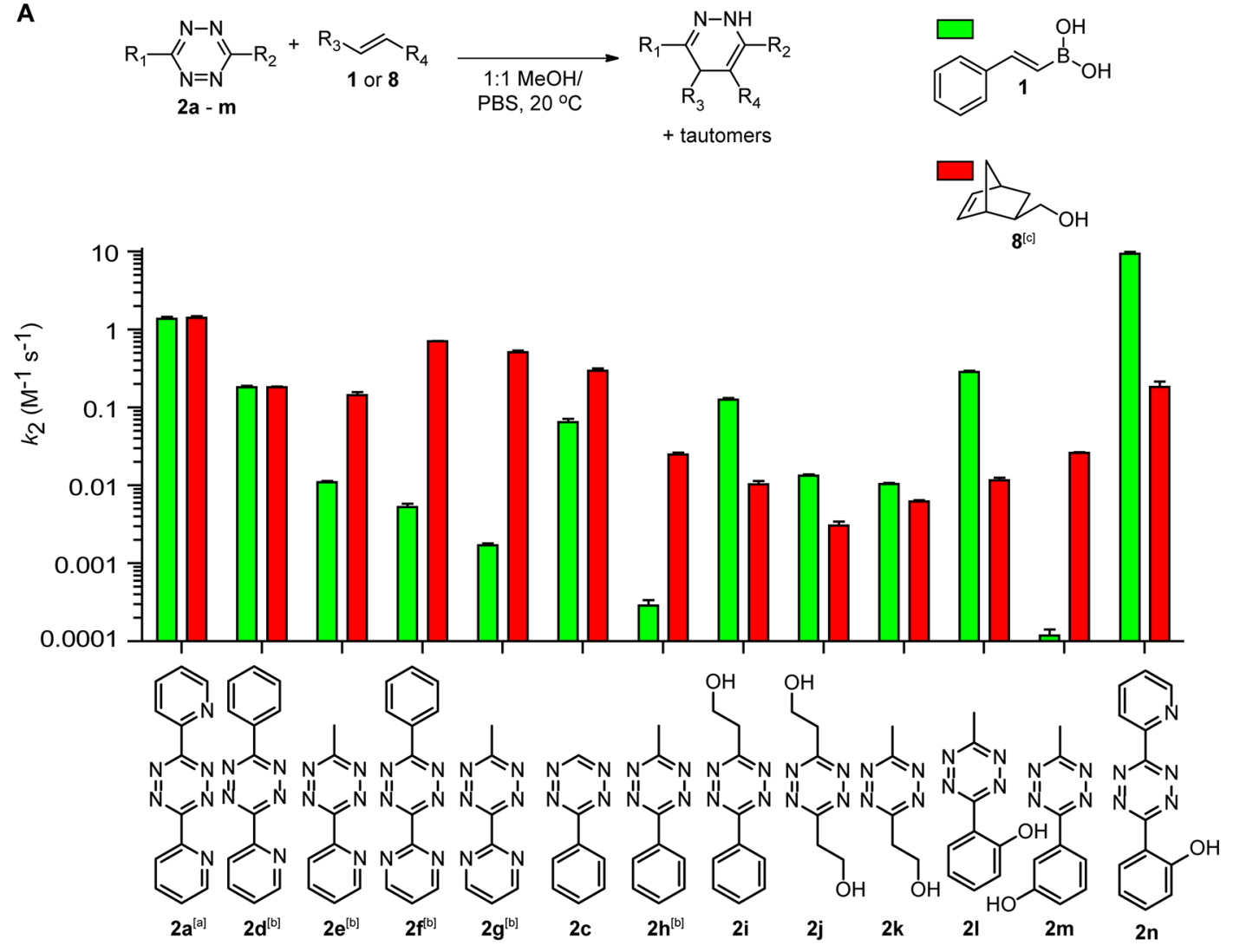

B

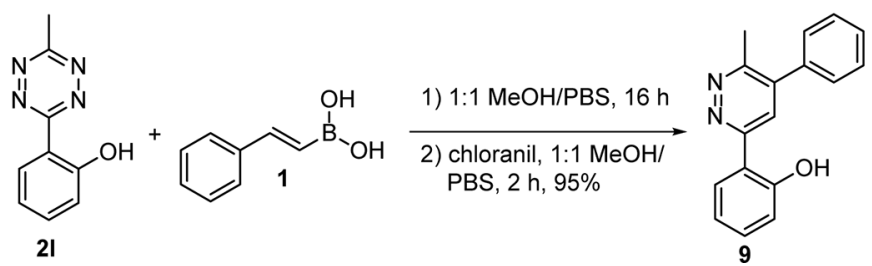

Figure 4. (A) Second-order rate constants of tetrazines $\mathbf{2 a}, \mathbf{c}-\mathbf{n}$ with VBA 1 and norbornene 8 measured in $1: 1 \mathrm{MeOH} / \mathrm{PBS}(\mathrm{pH}=7.4)$ at $20{ }^{\circ} \mathrm{C}$, shown on a logarithmic scale. (B) Product formation of the reaction of tetrazine 21 with VBA 1 and subsequent oxidation with chloranil, yielding pyridazine 9 as a single isomer. [a] Previously reported. ${ }^{18}[\mathrm{~b}]$ Previously reported. ${ }^{21}[\mathrm{c}]$ Endo/exo $=2: 1$.

the tetrazine for stacking. Norbornene $\mathbf{8}$ gave a slightly lower $k_{2}$ value with both tetrazines $\mathbf{2 j}$ and $\mathbf{2 k}$ compared to the more electron deficient tetrazine $\mathbf{2 e}$, as expected.

Next, we investigated tetrazines bearing a hydroxyl substituent on the phenyl ring in the reaction with VBA and norbornene. The $o$-hydroxyphenyl methyl tetrazine 21 gave a high rate constant of $0.28 \mathrm{M}^{-1} \mathrm{~s}^{-1}$ for VBA 1, almost 1000-fold higher than the $k_{2}$ of phenyl methyl tetrazine $2 \mathbf{h}$. Moreover, the reactivity of $\mathbf{2 l}$ with norbornene $\mathbf{8}$ was 23 -fold lower, making this tetrazine more selective for VBA. In contrast, $m$ hydroxyphenyl tetrazine $2 \mathrm{~m}$ gave a more than 3 orders of magnitude decrease in reaction rate compared to the ohydroxy-substituted 2l, possibly due to unfavorable positioning of the hydroxyl for coordination. Important to emphasize is that the hydroxyl substitution pattern is not relevant for the tetrazine ligations with norbornene, as $m$-hydroxy-substituted tetrazine $\mathbf{2 m}$ gave a comparable rate constant as the $o$-hydroxysubstituted tetrazine 21. Furthermore, $o$-hydroxyphenyl pyridyl tetrazine $2 \mathrm{~m}$ additionally increased the reaction rate more than 30-fold to $9.3 \mathrm{M}^{-1} \mathrm{~s}^{-1}$ for VBA and thereby exceeding the rate constant of dipyridyl-s-tetrazine $2 \mathrm{a}$ with about 1 order of magnitude. We additionally attempted to synthesize and measure the rate constants for disubstituted o-hydroxyphenyl tetrazine; however, the insolubility of this tetrazine in methanol unfortunately hampered our measurements.

The results above indicates that coordination of a hydroxylsubstituent on the tetrazine to the boronic acid has a positive influence on the rate of the iEDDA cycloaddition. Furthermore, the hydroxyl-substituted tetrazines are much more selective for VBA than for norbornene. Whereas dipyridyl-s-tetrazine $\mathbf{2 a}$ gives comparable rate constants for both alkenes, tetrazine 2 gives, for example, a 52 -fold higher $k_{2}$ for VBA 1 than for norbornene 8. To validate that the increase in reactivity of the VBAs toward the hydroxylsubstituted tetrazines is indeed caused by coordination, we isolated the product of VBA 1 and tetrazine 21 (Figure 4B). Since the reaction of VBA 1 with tetrazine 21 gave several tautomers of the dihydropyridazine, we oxidized the product to the corresponding pyridazine to facilitate characterization of the product. This two-step reaction gave pyridazine 9 as a single isomer in nearly quantitative yield with the phenyl substituent on the 5-position of the pyridazine ring, demonstrating the coordination of the boronic acid to the $o$ hydroxyphenyl ring of tetrazine 21 . The reaction of VBA 1 with 


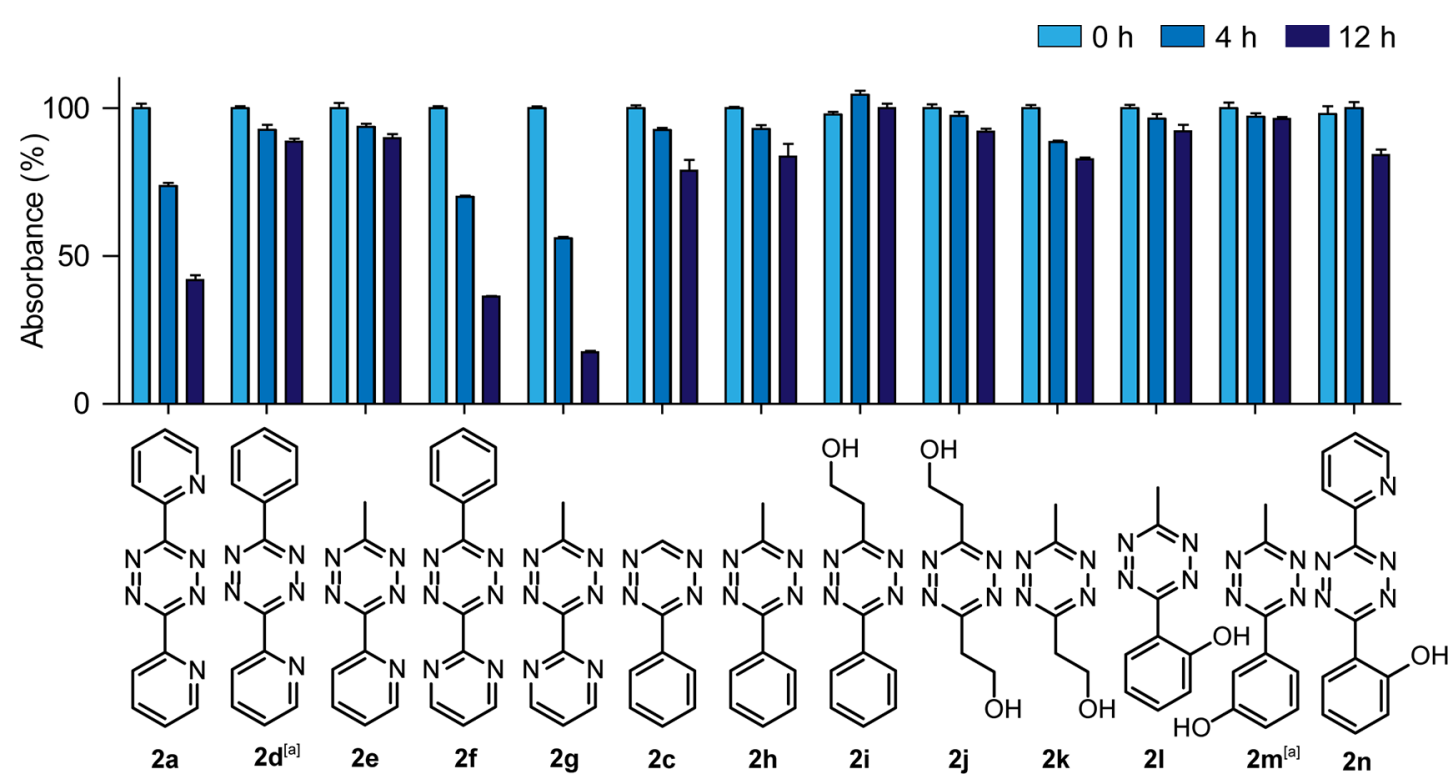

Figure 5. Decrease of absorbance at $540 \mathrm{~nm}$ of tetrazines $\mathbf{2 a}, \mathbf{c}-\mathbf{n}$ in $1: 9 \mathrm{DMSO} / \mathrm{PBS}(\mathrm{pH}=7.4)$ at $37^{\circ} \mathrm{C}$. The normalized mean with SD is plotted. [a] Measured in 1:1 DMSO/PBS $(\mathrm{pH}=7.4)$ as tetrazines $\mathbf{2 d}$ and $\mathbf{2 m}$ were insoluble in 1:9 DMSO/PBS.

tetrazine $2 \mathrm{n}$ also resulted in a single product after oxidation; however, the exact regioisomer could not be established due to the absence of a clear NOESY signal in $2 \mathrm{D}{ }^{1} \mathrm{H}$ NMR.

Stability of Tetrazines in Aqueous Environment. The bioorthogonal application of tetrazines requires that these moieties are stable in aqueous solution or biological environment. However, some tetrazines slowly decompose in aqueous environment with especially electron-withdrawing substituents destabilizing the aromatic ring. ${ }^{28,29}$ As we expect that the hydroxyl-containing tetrazines described above are more electron-rich than the pyridyl- or pyrimidyl-substituted tetrazines, we predicted that the former also have superior stability. Therefore, we tested the stability of tetrazines $\mathbf{2 a}, \mathbf{c}-\mathbf{n}$ in aqueous environment at $37^{\circ} \mathrm{C}$ by measuring the decrease in absorbance of the tetrazines at $540 \mathrm{~nm}$ (Figure 5, Table S2).

As expected, we observed that dipyridyl-s-tetrazines $2 \mathbf{a}$ as well as pyrimidyl-substituted tetrazines $\mathbf{2} \mathbf{f}$ and $\mathbf{2} \mathbf{g}$ were rather unstable, with $60-85 \%$ of the tetrazines being degraded after $12 \mathrm{~h}$ in 1:9 DMSO/PBS. In contrast, the more electron-rich pyridyl tetrazines $\mathbf{2 d}$ and $2 \mathrm{e}$ and phenyl tetrazines $\mathbf{2 c}$ and $\mathbf{2 h}$ were more stable, with at least $75 \%$ of the tetrazines remaining after 12 h. To our delight, all hydroxyl-substituted tetrazines $\mathbf{2} \mathbf{i}-\mathbf{n}$ were similarly stable and only marginal degradation was visible after $12 \mathrm{~h}$.

\section{CONCLUSIONS}

To conclude, we set out here to gain more insight in the notable reactivity of VBAs toward tetrazines containing a pyridine substituent, of which the nitrogen coordinates to the boronic acid of the VBA. We compared the reactivity of vinylboronic acids to their negatively charged boronate anion in the tetrazine ligation using DFT calculations and an experimental setup, where we changed the $\mathrm{pH}$ of the reaction buffer, and observed that the more electron-rich boronate anion gave a faster reaction with tetrazines. We synthesized several hydroxyl-substituted tetrazines and showed that the hydroxyl substituent coordinates to the boronic acid and increases the rate constants of the tetrazine ligation with VBAs of several orders of magnitude. Moreover, these new tetrazines were more electron-rich than the pyridyl-substituted tetrazines giving lower rate constants with norbornene and favoring reactivity toward VBA due to coordination. Furthermore, the hydroxyl-substituted tetrazines were found to be more stable in aqueous media and therefore more suitable for a bioorthogonal application requiring long incubation times compared to the pyridyl-substituted tetrazines. The developed hydroxyl-substituted tetrazines are a valuable asset for bioorthogonal conjugation due to their small size, stability, and hydrophilic character. Furthermore, the selectivity of the hydroxyl tetrazines toward VBAs renders them potential candidates for use in orthogonal bioorthogonal tetrazine ligations for dual labeling of biomolecules, when combined with the reaction of a strained alkene and a tetrazine lacking a boron-coordinating substituent.

\section{ASSOCIATED CONTENT}

\section{S Supporting Information}

The Supporting Information is available free of charge on the ACS Publications website at DOI: 10.1021/acs.bioconjchem. 8 b00439.

Experimental details, full spectroscopic data for all new compounds, Cartesian coordinates of all species occurring in our model DFT computations, kinetics for rate constants determination, and additional figures (PDF)

\section{AUTHOR INFORMATION}

\section{Corresponding Authors}

*E-mail: f.m.bickelhaupt@vu.nl.

*E-mail: k.bonger@science.ru.nl.

ORCID $\odot$

Anthonius H. J. Engwerda: 0000-0001-9589-7129

F. Matthias Bickelhaupt: 0000-0003-4655-7747

Kimberly M. Bonger: 0000-0001-9498-2620

Notes

The authors declare no competing financial interest. 


\section{ACKNOWLEDGMENTS}

Selma Eising and Kimberly M. Bonger thank The Netherlands Research Institute for Chemical Biology (NRSCB) and the Institute of Molecules and Materials (IMM) of the Radboud University in Nijmegen for financial support. F. Matthias Bickelhaupt acknowledges financial support from The Netherlands Organization for Scientific Research (NWO).

\section{REFERENCES}

(1) Shih, H.-W., Kamber, D. N., and Prescher, J. A. (2014) Building better bioorthogonal reactions. Curr. Opin. Chem. Biol. 21, 103-111.

(2) Shieh, P., and Bertozzi, C. R. (2014) Design Strategies for Bioorthogonal Smart Probes. Org. Biomol. Chem. 12, 9307-9320.

(3) King, M., and Wagner, A. (2014) Developments in the field of bioorthogonal bond forming reactions - past and present trends. Bioconjugate Chem. 25, 825-839.

(4) Chen, X., and Wu, Y.-W. (2016) Selective chemical labeling of proteins. Org. Biomol. Chem. 14, 5417-5439.

(5) Šečkutè, J., and Devaraj, N. K. (2013) Expanding room for tetrazine ligations in the in vivo chemistry toolbox. Curr. Opin. Chem. Biol. 17, 761-767.

(6) Knall, A.-C., and Slugovc, C. (2013) Inverse electron demand Diels-Alder (iEDDA)-initiated conjugation: a (high) potential click chemistry scheme. Chem. Soc. Rev. 42, 5131-5142.

(7) Kozma, E., Demeter, O., and Kele, P. (2017) Bioorthogonal Fluorescent Labelling of Biopolymers via Inverse Electron Demand Diels-Alder Reactions. ChemBioChem 18, 486-501.

(8) Oliveira, B. L., Guo, Z., and Bernardes, G. J. L. (2017) Inverse electron demand Diels-Alder reactions in chemical biology. Chem. Soc. Rev. 46, 4895-4950.

(9) Borrmann, A., Milles, S., Plass, T., Dommerholt, J., Verkade, J. M. M., Wiessler, M., Schultz, C., van Hest, J. C. M., van Delft, F. L., and Lemke, E. A. (2012) Genetic Encoding of a Bicyclo[6.1.0] nonyne-Charged Amino Acid Enables Fast Cellular Protein Imaging by Metal-Free Ligation. ChemBioChem 13, 2094-2099.

(10) Lang, K., Davis, L., Wallace, S., Mahesh, M., Cox, D. J., Blackman, M. L., Fox, J. M., and Chin, J. W. (2012) Genetic encoding of bicyclononynes and trans-cyclooctenes for site-specific protein labeling in vitro and in live mammalian cells via rapid fluorogenic diels-alder reactions. J. Am. Chem. Soc. 134, 10317-10320.

(11) Blackman, M. L., Royzen, M., and Fox, J. M. (2008) Tetrazine Ligation: Fast Bioconjugation Based on Inverse-Electron-Demand Diels-Alder Reactivity. J. Am. Chem. Soc. 130, 13518-13519.

(12) Selvaraj, R., and Fox, J. M. (2013) trans-cyclooctene - a stable, voracious dienophile for bioorthogonal labeling. Curr. Opin. Chem. Biol. 17, 753-760.

(13) Devaraj, N. K., Weissleder, R., and Hilderbrand, S. A. (2008) Tetrazine-based cycloadditions: application to pretargeted live cell imaging. Bioconjugate Chem. 19, 2297-2299.

(14) Patterson, D. M., Nazarova, L. A., Xie, B., Kamber, D. N., and Prescher, J. A. (2012) Functionalized cyclopropenes as bioorthogonal chemical reporters. J. Am. Chem. Soc. 134, 18638-18643.

(15) Yang, J., Šečkute, J., Cole, C. M., and Devaraj, N. K. (2012) Live-cell imaging of cyclopropene tags with fluorogenic tetrazine cycloadditions. Angew. Chem., Int. Ed. 51, 7476-7479.

(16) Niederwieser, A., Späte, A.-K., Nguyen, L. D., Jüngst, C., Reutter, W., and Wittmann, V. (2013) Two-color glycan labeling of live cells by a combination of diels-alder and click chemistry. Angew. Chem., Int. Ed. 52, 4265-4268.

(17) Lee, Y.-J., Kurra, Y., Yang, Y., Torres-Kolbus, J., Deiters, A., and Liu, W. R. (2014) Genetically encoded unstrained olefins for live cell labelling with tetrazine dyes. Chem. Commun. 50, 13085-13088.

(18) Eising, S., Lelivelt, F., and Bonger, K. M. (2016) Vinylboronic Acids as Fast Reacting, Synthetically Accessible, and Stable Bioorthogonal Reactants in the Carboni-Lindsey Reaction. Angew. Chem., Int. Ed. 55, 12243-12247.

(19) Eising, S., van der Linden, N. G. A., Kleinpenning, F., and Bonger, K. M. (2018) Vinylboronic Acids as Efficient Bioorthogonal
Reactant for Tetrazine Labeling in Living Cells. Bioconjugate Chem. 29, 982-986.

(20) Hall, D. G. (2011) Boronic acids, Preparation and Applications in Organic Synthesis, Medicine and Materials, WILEY-VCH, Weinheim.

(21) Eising, S., Xin, B. T., Kleinpenning, F., Heming, J., Florea, B. I., Overkleeft, H. S., and Bonger, K. M. (2018) Coordination-Assisted Bioorthogonal Chemistry: Orthogonal Tetrazine Ligations with Vinylboronic Acid and a Strained Alkene. ChemBioChem 19, 1648.

(22) te Velde, G., Bickelhaupt, F. M., Baerends, E. J., Fonseca Guerra, C., van Gisbergen, S. J. A., Snijders, J. G., and and Ziegler, T. (2001) Chemistry with ADF. J. Comput. Chem. 22, 931-967.

(23) Baker, J., and Pulay, P. (2002) Assessment of the Handy-Cohen optimized exchange density functional for organic reactions. J. Chem. Phys. 117, 1441-1449.

(24) Handy, N. C., and Cohen, A. J. (2001) Left-right correlation energy. Mol. Phys. 99, 403-412.

(25) Lee, C., Yang, W., and Parr, R. G. (1988) Development of the Colle-Salvetti correlation-energy formula into a functional of the electron density. Phys. Rev. B: Condens. Matter Mater. Phys. 37, 785789.

(26) Bickelhaupt, F. M., and Houk, K. N. (2017) Analyzing Reaction Rates with the Distortion/Interaction-Activation Strain Model. Angew. Chem., Int. Ed. 56, 10070-10086.

(27) Bickelhaupt, F. M., and Baerends, E. J. (2000) Reviews in Computational Chemistry (Lipkowitz, K. B., and Boyd, D. B.) Wiley$\mathrm{VCH}$, New York.

(28) Versteegen, R. M., Rossin, R., Ten Hoeve, W., Janssen, H. M., and Robillard, M. S. (2013) Click to release: Instantaneous doxorubicin elimination upon tetrazine ligation. Angew. Chem., Int. Ed. 52, 14112-14116.

(29) Karver, M. R., Weissleder, R., and Hilderbrand, S. A. (2011) Synthesis and evaluation of a series of 1,2,4,5-tetrazines for bioorthogonal conjugation. Bioconjugate Chem. 22, 2263-2270. 